\title{
加压烧结合成 $\mathrm{HgBa}_{2} \mathrm{Ca}_{2} \mathrm{Cu}_{3} \mathrm{O}_{\mathbf{8}+\delta}$ 超导体 的研究 ${ }^{*}$
}

\author{
郡惠民 ${ }^{(1)}$ 沈临江 (2) 吴诤 (2) 李贞俊 ${ }^{(2)}$ 袁丕方 $^{\text {(2) }}$ \\ 蔡永明 (2 张共宁 (2) 姚希贤 ${ }^{(1)}$
}

(1) 南京大学物理系固体微结构实验室, 南京 210093; (2) 南京化工学院超导实验室, 南京 210009)

\section{关链词 $\mathrm{Hg}$ 系超导体 $\mathrm{HgBa}_{2} \mathrm{Ca}_{2} \mathrm{Cu}_{3} \mathrm{O}_{8+\delta}$ 加压烧结}

自 Putilin 和 Schilling 等人发现了 $\mathrm{Hg}-\mathrm{Ba}-\mathrm{Ca}-\mathrm{Cu}-\mathrm{O}$ 系统的 $T_{\mathrm{c}}$ 可达 $130 \mathrm{~K}$ 以上的超导性以 来 $^{[1,2]}$, 世界上拆起了一个研究 $\mathrm{Hg}$ 系超导体的高潮, 这不仅因为它创造了 $T_{\mathrm{c}}$ 的最高记录, 而更 重要的是该化合物系具有值得深人研究的、十分有趣的物理特性及超导机制. 然而, 由于 $\mathrm{HgO}$ 的熔点低、易挥发、且毒性大, 所以造成制备这一系统的超导样品的难度相当大. 目前通常采 用两种方法来制备该系统的超导样品: (1) 高压法 ${ }^{[3]}$; (2) 封管法 ${ }^{[4]}$. 前者由于压力太高 (一般 $40 \times 10^{5} \mathrm{kPa}$ 以上), 所以一般实验室难以实现; 后者由于抽空石英管内 $\mathrm{HgO}$ 的分解, 往往会造成很 高的 $\mathrm{Hg}$ 蒸汽压一一几巴, 甚至达 $100 \times 10^{5} \mathrm{~Pa}$ 以上 ${ }^{[\mathrm{g}}$. 常有爆炸发生, 难以重复制备. 因此需 要一个普通实验室就能实现的、操作易行的制备 $\mathrm{Hg}$ 系超导化合物的方法.

本文报道我们在不太高的压力下 $\left(100 \times 1.01 \times 10^{5} \mathrm{~Pa} \sim 300 \times 1.01 \times 10^{5} \mathrm{~Pa}\right)$ 烧结, 成功地 合成了 $\mathrm{HgBa}_{2} \mathrm{Ca}_{2} \mathrm{Cu}_{3} \mathrm{O}_{8+\delta}$ 超导体的研究结果. 这一方法, 既保持了 “封管法” 的加压烧结合成 条件, 又克服了 “封管法”易爆炸的弱点; 另一方面, 它又不需要 “高压法” 中所用的庞大高压设 备.

\section{1 实验}

由分析纯的金属氧化物粉末 $\mathrm{HgO}, \mathrm{BaO}, \mathrm{CaO}$ 和 $\mathrm{CuO}$ 为原料, 以名义组成 $\mathrm{HgBa}_{2} \mathrm{Ca}_{2} \mathrm{Cu}_{3} \mathrm{O}_{8+\delta}$ 按适当比例称料, 混合、压片, 再经短时热处理 $\left(725^{\circ} \mathrm{C}\right.$ 烧结, 历时 $3 \sim 4 \mathrm{~min}$, 重复二次以上) 后 ${ }^{[6}$, 包银密封, 放人特制的加压烧结炉 (如图 1 所示) 内加压烧结. 以 $30 \sim 35^{\circ} \mathrm{C} / \mathrm{min}$ 速率升温, 在 $675 \sim 750^{\circ} \mathrm{C}$ 烧结 $1 \sim 1.5 \mathrm{~h}$, 然后溶火到室温, 接着在高压 $\left(120 \times 1.01 \times 10^{5} \mathrm{~Pa}\right)$ 氧 气中退火 $30 \mathrm{~h}$ 以上. 退火处理过的样品接着进行 $\mathrm{X}$-射线衍射分析, 交流磁化率及电阻等测 量.

\section{2 结果与讨论}

为了总结烧结效果, 我们在各种压力下 $\left(100 \times 1.01 \times 10^{5} \sim 300 \times 1.01 \times 10^{5} \mathrm{~Pa}\right)$ 进行了实

1994-06-22 收稿, 1995-01-15 收修改稿

* 国家超导研究和开发中心资助项目 
验, 实验结果表明, 增加压力对样品的起始转变温度影响不大, 但烧结温度可以适当 降低, 如果在压力 $200 \times 1.01 \times 10^{5} \mathrm{~Pa}$, 烧结温度为 $725^{\circ} \mathrm{C}$, 则实验结果较为理想. 所以, 以下 样品均在这个压力及温度下烧结而成.

在制备 $\mathrm{Hg}$ 系超导铜氧化物时, 能否阻止形成 $\mathrm{CaHgO}_{2}$ 产物, 是很重要的. 一旦在制备初 期形成了 $\mathrm{CaHgO}_{2}$, 它会阻止 $\mathrm{Hg}$ 系超导化合物的形成, 这也许是为什么在制备 $\mathrm{Hg}$ 系超导样 品时需要采用高压高温的原因. 相反地, 如果在制备中避免形成 $\mathrm{CaHgO}_{2}$ 化合物, 用金属氧化 物的粉末为原料, 则在所需条件下很快形成了 $\mathrm{Hg}$ 系超导铜氧化物, 短则十几分钟就可形成.

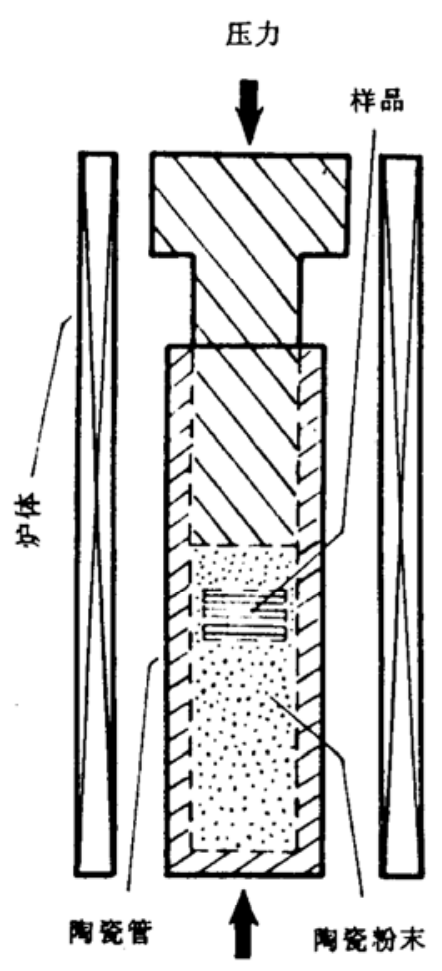

图 1 加压烧结炉示意图

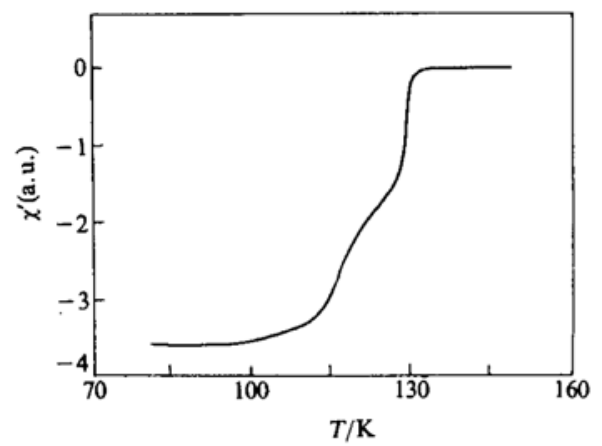

图 $4 \mathrm{HgBa}_{2} \mathrm{Ca}_{2} \mathrm{Cu}_{3} \mathrm{O}_{8+\delta}$ 样品的交流磁化率随 温度变化的曲线 从曲线可知抗磁变温度为 $132.4 \mathrm{~K}$

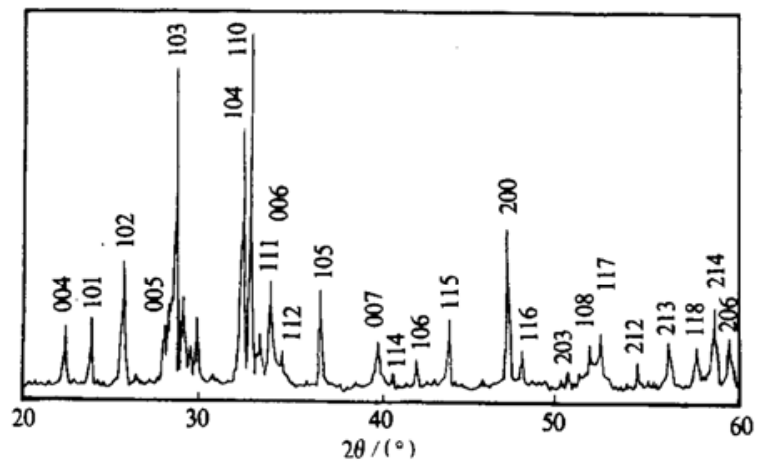

图 2 加压合成的 $\mathrm{HgBa}_{2} \mathrm{Ca}_{2} \mathrm{Cu}_{3} \mathrm{O}_{8+\delta}$ 样品的 $\mathrm{X}$ 射线衍射图 晶体属四方晶系, 晶胞能参数经最小二乘法修正后为 $a=0.385 \mathrm{~nm}$ 和 $c=1.578 \mathrm{~nm}$

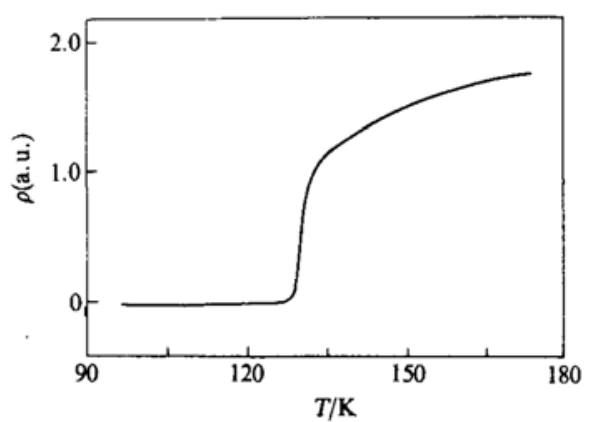

图 $3 \mathrm{HgBa}_{2} \mathrm{Ca}_{2} \mathrm{Cu}_{3} \mathrm{O}_{8+\delta}$ 样品的电阻随温度变化的曲线 电阻温度为 $127 \mathrm{~K}$

实践证明, 我们使用的加压烧结炉的样品室的 密封状况相当好, 所以它创造了与 “封管法”同样的 烧结条件. 从而我们能成功地合成 $\mathrm{Hg}-1223$ 样品, 这 些也可以从图 2、图 3 和图 4 的实验结果看出来. 从图 2 所示的 XRD 衍射图可知, 所合成样品的主 相是 $\mathrm{Hg}-1223$ 相. 从图 3 和图 4 所示的曲线也可得到 同样的结论.

本实验成功的关键是: 加压烧结制备样品, 样品 室的密封程度是至关重要的; 此外, 烧结时间也不能 
太长。

\section{参考文献}

1 Putilin S N, Autipov E V, Chmaisson O et al. Superoonductivity at $94 \mathrm{~K}$ in $\mathrm{HgBa}_{2} \mathrm{CuO}_{4+\delta}$. Nature (London), 1993, 362: 226

2 Schilling A, Cantoni M, Guo J D et al. Superconductivity above $130 \mathrm{~K}$ in the $\mathrm{Hg}-\mathrm{Ba}-\mathrm{Ca}-\mathrm{Cu}-\mathrm{O}$ system Natue (London), 1993, 363: 56 58

3 Hirabayashi $\mathrm{M}$, Tokiwa $\mathrm{K}$, Tokumoto $\mathrm{M}$ et al. High-pressure synthesis of $\mathrm{HgBa}_{2} \mathrm{Ca}_{2} \mathrm{Cu}_{3} \mathrm{O}_{9-x}$ superconductor with $T_{\mathrm{c}}$ $>130$ K. Japan J Appl Phys, 1993, 32:1 206

$4 \mathrm{Zhu}$ W J, Huang Y Z, Chen L Q et al. Synthesis of the superconductors $\mathrm{HgBa}_{2} \mathrm{CaCu}_{2} \mathrm{O}_{6+\delta}$ and $\mathrm{HgBa}_{2} \mathrm{Ca}_{2} \mathrm{Cu}_{3} \mathrm{O}_{8+\delta}$. Physica, C, 1993, 218:5 7

5 Klehe A K, Gangopadhyay A K, Diederichs $\mathrm{J}$ et al. Dependenœe of the superconducting transition temperature of $\mathrm{HgBa}_{2} \mathrm{CuO}_{4+\delta}$ on hydrostatic pressure. Physica, C, 1993, 213:266 270

6 Shao $\mathrm{H} \mathrm{M}$, Shen L J, Shen J C et al. Synthesis of single-phase $\mathrm{HgBa}_{2} \mathrm{Ca}_{2} \mathrm{Cu}_{3} \mathrm{O}_{8+\delta}$ superoonductor. Physica, C, 1994, 232:5 9 